\title{
Correspondence
}

\section{Anaesthetic simulation}

To the Editor:

We appreciate the editorial "Anaesthetic simulation and lessons to be learned from aviation" by Drs. Helmreich and Davies ${ }^{1}$ published in the September issue of the Canadian Journal of Anaesthesia and offer the following comments:

Helmreich and Davies go into a lengthy discussion of content and ask: "How can the authors reassure us that these are indeed the right things to do and not just "clinically reasonable "without some form of validation, other than that described in this article?"

The concept of test validity is entirely different from test reliability and was not the purpose of our study. ${ }^{2}$ Our study did not evaluate the content or the validity of the simulator testing process whatsoever. Furthermore, our scenarios were designed to address the controversial proposed role of the simulator to assess the ability of new trainees and practicing clinicians to practice clinical skills and not to evaluate Crisis Management Skills.

Helmreich and Davies describe the evaluation and assessment process in the aviation industry in detail and claim: "Devitt et al. are using a medium to bigh fidelity simulator to provide part-task training to individual anaesthetists. "...In contrast, true CRM addresses teaminterface issues...".

Their description of the aviation industry Crew Resource Management (CRM) is interesting but bears little relevance to our published results. Our project to evaluate the simulator as a potential assessment tool is in no way connected to the ongoing Crisis Management Training Program (which actually does use teams including nurses and surgeons since 1995). ${ }^{3}$

Matt M. Kurrek MD

Hugh Devitt MD

Canadian Simulation Centre for Human

Performance and Crisis Management Training

Sunnybrook Health Science Centre

University of Toronto

\section{REFERENCES}

1 Helmreich $R L$, Davies JM. Anaesthetic simulation and lessons to be learned from aviation. Can J Anaesth 1997; 44: 907-12.

2 Devitt JH, Kurrek MM, Coben MM, et al. Testing the raters: inter-rater reliability of standardized anaesthesia simulator performance. Can J Anaesth 1997; 44: 924-8.
3 Anaesthesia Patient Safety Foundation (APSF)

Newletter Fall 1995.

(http://gasnet.med.yale.edu/apsf/newsletter/1995/

fall/simulator.html)

\section{REPLY}

We thank Drs. Kurrek and Devitt for their response to our editorial. We feel that our editorial reflects our concern with the direction described for the use of simulators in anaesthesia.

\section{R.L. Helmreich PhD}

University of Texas Aerospace Crew Research Center Austin, Texas, USA

J.M. Davies MD, FRCPC

Foothills Medical Centre

Calgary, Alberta

\section{Metabolic events with spontaneous malignant hyperthermia}

To the Editor:

In their very interesting paper (Kozak-Ribbens G. et al., "Metabolic events with spontaneous malignant hyperthermia...," 1997: 44: 757-64), the authors describe an $\mathrm{MH}$ episode that begins during recovery from one of several periods of electrical stimulation, and characterise this as spontaneous $\mathrm{MH}$ during non-triggering anaesthesia. But, electrical stimulation per se does indeed trigger $\mathrm{MH}$ episodes in susceptible pigs during nontriggering anaesthesia. ${ }^{l}$ Further, as with carbachol, the effect of electrical stimulation may be a direct acetylcholine-type arousal of skeletal muscle. which is exaggerated in susceptible skeletal muscle. ${ }^{2}$ Sympathetic arousal related to stimulation is unlikely to be a direct factor, ${ }^{3,4}$ although any increase in temperature related to sympathetic factors or to triggering may help to further advance the $\mathrm{MH}$ event. ${ }^{2,5,6}$

The authors elegantly document the intracellular acidosis associated with $\mathrm{MH}$ within skeletal muscle and amplify our information about the gradients between muscle and blood. As ionized lactate ion diffuses poorly out of the cell, the intracellular milieu, due to the MH episode, is more acidotic than is reflected by arterial blood gas analysis. ${ }^{7}$ As to hepatic temperature being greater than that in skeletal muscle, it is likely that the very high liver blood flow is, in part, 